\title{
Potential Role of Bordetella Pertussis in Celiac Disease
}

\author{
Keith Rubin, Steven Glazer ${ }^{*}$ \\ ILiAD Biotechnologies LLC, New York City, 10003 United States of America \\ *Corresponding author: glazer@iliadbio.com
}

Received March 17, 2015; Revised March 24, 2015; Accepted March 27, 2015

\begin{abstract}
We discuss the correlation between the incidence of acute clinical Bordetella pertussis infection and celiac disease in children $<2$ years of age during the Swedish celiac disease epidemic of the 1980s and 1990s.
\end{abstract}

Keywords: celiac disease, Bordetella pertussis, subclinical Bordetella pertussis colonization (SCBPC)

Cite This Article: Keith Rubin, and Steven Glazer, "Potential Role of Bordetella Pertussis in Celiac Disease.” International Journal of Celiac Disease, vol. 3, no. 2 (2015): 75-76. doi: 10.12691/ijcd-3-2-2.

\section{Introduction}

Marked increases in celiac disease (CD) prevalence in recent decades have expanded investigation into potential environmental causes and underlying mechanisms of CD. A 2014 review examined the challenges to establishing a rodent model for $\mathrm{CD}$, highlighting methods to overcome immunologic tolerance to oral gluten, including the use of bacterial adjuvants [1]. Bordetella pertussis toxin $\left(\mathrm{BP}_{\mathrm{TOX}}\right)$ is one such adjuvant used in many animal models of human autoimmune and allergic disease [1,2,3], including CD-associated dermatitis herpetiformis, and intraperitoneal $\mathrm{BP}_{\text {Tox }}$-mediated gluten sensitization reproduces $\mathrm{CD}$ markers [1]. Interestingly, animal modeling demonstrates that "nanogram quantities of PT [pertussis toxin, $\mathrm{BP}_{\mathrm{TOX}}$ ], when administered with a food protein, result in longterm sensitization to the antigen" in the small intestine [3].

Mindful of the adjuvanticity of $\mathrm{BP}_{\mathrm{TOx}}$, we note the concurrent decades-long rise in rates of CD [4] and Bordetella pertussis (BP) in the United States, the latter ascribed to multiple factors, including the inability of acellular pertussis (aP) vaccination to prevent subclinical BP colonization (SCBPC) [5]. Recent human data confirm nasopharyngeal SCBPC by polymerase chain reaction in $4.8 \%$ of asymptomatic children in a highly (99\%) aPvaccinated population [6]. Given the potential biologic effects of SCBPC, we compared the rates of acute BP and $\mathrm{CD}$ in children $<2$ years of age during the Swedish CD epidemic of the 1980s and 1990s.

\section{Discussion}

Figure 1 presents the concurrence of acute BP (whooping cough) and CD during the Swedish CD epidemic, most notable for simultaneous and rapid declines associated with the reintroduction of pertussis vaccination after a 15 year national hiatus. Most Swedish children received whole cell BP vaccination from the 1950s to 1979, when it was suspended for efficacy and safety concerns [7]. While mean CD incidence subsequently increased, infant CD ascertainment was likely limited prior to the emergence of serum IgA-based screening tests in 1983-1984 [8]. Such blood-based screening rapidly gained acceptance with consideration in European practice guidelines by 1990 [8].

As seen in Figure 1, covariation between BP and infant CD is evident from 1986 to 2000, peaking together in 1994.

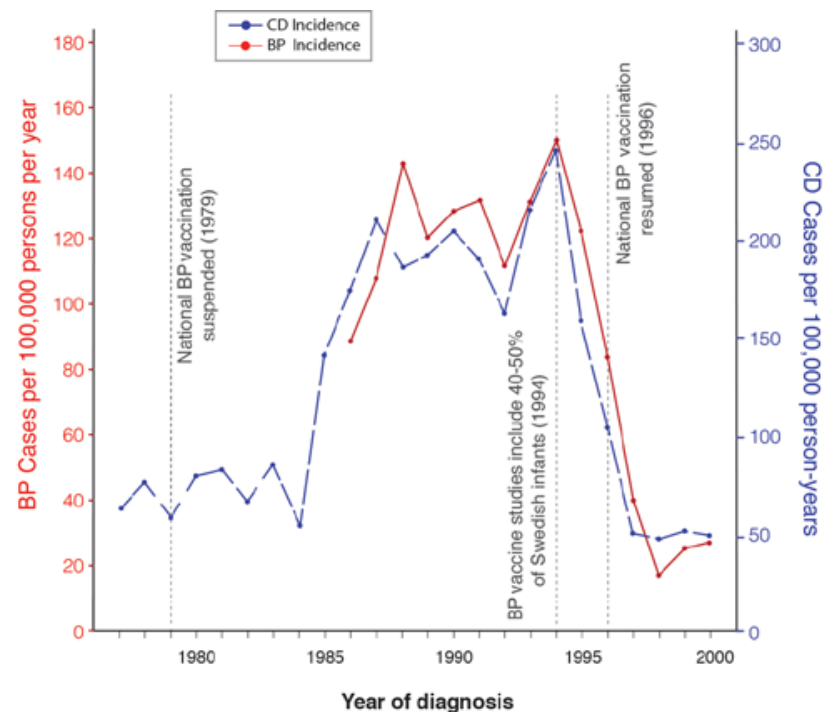

Figure 1. Concurrent Swedish Pertussis and Celiac Disease Epidemics, 1986-2000. The cessation of BP immunization in Sweden in 1979 preceded a rise in BP in the 1980s, persisting until acellular BP vaccination trials in 1993-1994 and broad implementation of vaccination in 1996 (red line) [7]. The incidence of CD in Swedish children < 2 years of age, based on data from Olsson et al. (dashed blue line) [9], closely tracks BP incidence in Sweden during this period.

The resurgence of BP prompted aP vaccination trials by 1993-1994, immunizing 40-50\% of Swedish infants [7]. Abrupt proportionate decreases in BP and CD followed. In 1996, nation-wide aP vaccination resumed, reinforcing the drop in BP rates. Of the many Swedish childhood vaccination programs tracked from 1980-2000, the only disease for which vaccine coverage increased as infant CD declined in the 1990s, was BP [10]. 
Despite the association between acute BP and CD incidence, no association was found between aP vaccination and $\mathrm{CD}$ in the same child [10]. Since aP vaccination directly protects against acute $\mathrm{BP}$ but not against $\mathrm{CD}$, acute $\mathrm{BP}$ must have covaried with another cause of CD. We propose that acute BP and SCBPC carry distinct disease risk profiles. As aP vaccination fails to prevent SCBPC in primates [5], and SCBPC persists in highly aP-vaccinated human populations [6], we propose that SCBPC covaried with acute BP and CD in Sweden, and is an unrecognized cause of CD. We further submit that reintroduction of aP vaccination in the 1990s reduced SCBPC indirectly, by decreasing ambient BP exposure and seeding of SCBPC. The role of nasopharyngeal SCBPC-mediated sensitization in CD animal models, and more broadly, in allergic and autoimmune human disease merits further investigation.

\section{Conclusion}

Given the covariation between BP and CD in Sweden, that $\mathrm{BP}$ and $\mathrm{BP}_{\mathrm{TOx}}$ are potent adjuvants, that SCBPC secretes $\mathrm{BP}_{\mathrm{TOx}}$ and is common in highly aP-vaccinated populations, that intraperitoneal $\mathrm{BP}_{\mathrm{TOX}}$-mediated gluten sensitization (without colocalization) reproduces CD markers, and that minute quantities of $\mathrm{BP}_{\mathrm{TOx}}$ administered with otherwise non-imunogenic food protein induce longterm antigen sensitization, it is biologically plausible that SCBPC-mediated mucosal sensitization to colocalized gliadin is a major unrecognized cause of CD.

\section{Statement of Competing Interests}

Drs. Rubin and Glazer are employed by ILiAD Biotechnologies LLC, which is developing a vaccine for the prevention of Bordetella pertussis.

\section{Abbreviations}

$\mathrm{BP}_{\mathrm{TOx}}$ : Bordetella pertussis toxin

aP: acellular pertussis

SCBPC: subclinical Bordetella pertussis colonization.

\section{References}

[1] Korneychuk, N., B. Meresse, and N. Cerf-Bensussan, Lessons from rodent models in celiac disease. Mucosal immunology, 2014.

[2] Chen, X., et al., Pertussis toxin as an adjuvant suppresses the number and function of CD4+ CD25+ $\mathrm{T}$ regulatory cells. European Journal of Immunology, 2006. 36(3): p. 671-680.

[3] Kosecka, U., M.C. Berin, and M.H. Perdue, Pertussis adjuvant prolongs intestinal hypersensitivity. International archives of allergy and immunology, 1999. 119(3): p. 205-11.

[4] Rubio-Tapia, A., et al., Increased prevalence and mortality in undiagnosed celiac disease. Gastroenterology, 2009. 137(1): p. 8893.

[5] Warfel, J.M., L.I. Zimmerman, and T.J. Merkel, Acellular pertussis vaccines protect against disease but fail to prevent infection and transmission in a nonhuman primate model. Proceedings of the National Academy of Sciences, 2014. 111(2): p 787-792.

[6] Zhang, Q., et al., Prevalence of asymptomatic Bordetella pertussis and Bordetella parapertussis infections among school children in China as determined by pooled real-time PCR: A cross-sectional study. Scandinavian journal of infectious diseases, 2014. 46(4): p. 280-287.

[7] Olin, P., et al., Declining pertussis incidence in Sweden following the introduction of acellular pertussis vaccine. Vaccine, 2003. 21(17-18): p. 2015-21.

[8] Waker-Smith, J., et al., Revised criteria for diagnosis of celiac disease. Report of Working Group of European Society of Pediatric Gastroenterology and Nutrition. Arch Dis Child, 1990. 65: p. 909-11.

[9] Olsson, C., et al., Difference in celiac disease risk between Swedish birth cohorts suggests an opportunity for primary prevention. Pediatrics, 2008. 122(3): p. 528-34.

[10] Myleus, A., et al., Early vaccinations are not risk factors for celiac disease. Pediatrics, 2012. 130(1): p. e63-70. 